\title{
THETA CONSTANTS AND CUSP FORMS
}

\author{
BY
}

\section{A. J. CRISALlI}

ABSTRACT. For principal congruence subgroups of levels 2 and 4 a basis for their cusp forms consisting of monomials of theta constants is displayed. Some conditions for the vanishing of Poincare series of these groups are found.

Introduction. In this paper we shall discuss a special case of a fundamental theorem of Igusa's [3, p. 235] which asserts that the integral closure of a graded ring of theta constants is the graded ring of modular forms for a certain subgroup $\Gamma\left(r^{2}, 2 r^{2}\right)$ of the modular group. The special case will be that of a single complex variable with $r=2$. We will obtain a basis for the space of cusp forms for $\Gamma(4,8)$ consisting of monomials of theta constants, and use this information to derive similar bases for $\Gamma(4)$ and $\Gamma(2)$. We will then obtain information about the vanishing of Poincare series for $\Gamma(4)$ and $\Gamma(2)$.

1. The group $\Gamma(n, 2 n)$. Let $\Gamma(1)$ denote the group of $2 \times 2$ matrices with rational integer coefficients and determinant 1 . If $n$ is a positive integer let $\Gamma(n)$ denote the principal congruence subgroup of level $n$, that is, the subgroup of $\Gamma(1)$ defined by the condition $M \equiv 1_{2} \bmod n$. Also, let $\Gamma(n, 2 n)$ denote the set of all matrices $M$ in $\Gamma(1)$ defined by the conditions $M \equiv 1_{2} \bmod$ $n, c d \equiv a b \equiv 0 \bmod 2 n$ where $M=\left(\begin{array}{ll}a & b \\ c & d\end{array}\right)$.

In $[2$, p. 222] it is proved that for $n$ an even positive integer, $\Gamma(n, 2 n)$ is a normal subgroup of $\Gamma(n)$ and its index in $\Gamma(n)$ is 4 .

If $G$ is any subgroup of $\Gamma(1)$ we shall denote $G^{\prime}$ the corresponding inhomogeneous group, that is, the group of linear fractional transformations $T(z)=(a z+b) /(c z+d)$ where $\left(\begin{array}{ll}a & b \\ c & d\end{array}\right)$ is in $G$. Thus, if $n=1$ or $n=2$ then $\Gamma(n)^{\prime} \cong \Gamma(n) /\left( \pm 1_{2}\right)$ and if $n>2$ then $\Gamma(n)^{\prime} \cong \Gamma(n)$. Again, if $n=1$ or $n=2$ then $\Gamma(n, 2 n)^{\prime} \cong \Gamma(n, 2 n) /\left( \pm 1_{2}\right)$ and if $n>2$ then $\Gamma(n, 2 n)^{\prime} \cong$ $\Gamma(n, 2 n)$. Thus for all positive even $n$

$$
\Gamma(n)^{\prime} / \Gamma(n, 2 n)^{\prime} \cong \Gamma(n) / \Gamma(n, 2 n)
$$

and $\Gamma(n, 2 n)^{\prime}$ is a normal subgroup of $\Gamma(n)^{\prime}$ of index 4 . Now the index of 
$\Gamma(n)^{\prime}$ in $\Gamma(1)^{\prime}$ is known to be $1 / 2 n^{3} \Pi\left(1-1 / p^{2}\right)$ where in the product $p$ runs through all primes dividing $n$. Thus the index of $\Gamma(n, 2 n)^{\prime}$ in $\Gamma(1)^{\prime}$ is

$$
\left[\Gamma(1)^{\prime}: \Gamma(n)^{\prime}\right]\left[\Gamma(n)^{\prime}: \Gamma(n, 2 n)^{\prime}\right]=2 n^{3} \Pi\left(1-1 / p^{2}\right) .
$$

It is known that the genus of the Riemann surface associated with a normal subgroup $G$ of index $h \geqslant 4$ in $\Gamma(1)^{\prime}$ is given by the formula genus $(G)=$ $1+h\left(n_{0}-6\right) / 12 n_{0}$ where $n_{0}$ is the smallest positive integer such that $z \Rightarrow$ $z+s$ is in $G$. For $G=\Gamma(n, 2 n)^{\prime}$ we see by its definition that $n_{0}=2 n$ and so we have established that

$$
\operatorname{genus}\left(\Gamma(n, 2 n)^{\prime}\right)=1+n^{2}(n-3) \Pi\left(1-1 / p^{2}\right) / 6 .
$$

2. Modular forms for $\Gamma(n, 2 n)$. Let $G$ be a subgroup of $\Gamma(1)$ of finite index and let $k$ be a nonnegative integer. By a modular form of weight $k$ for $G$ we mean a holomorphic function $f(z)$ in the upper half plane and at the parabolic vertices (cusps) of $G$ such that for all $M(z)$ in $G^{\prime}$ we have $f(M(z))=(c z+d)^{k} f(z)$. Let $A(G)_{k}$ denote the vector space over $\mathbf{C}$ of modular forms of weight $k$. By a cusp form of $G$ of weight $k$ we mean an element of $A(G)_{k}$ which vanishes at the parabolic vertices of $G$. Let $C(G)_{k}$ denote the subspace of cusp forms of weight $k$.

We now use a standard procedure to compute $\operatorname{Dim} A(G)_{k}$ and $\operatorname{Dim} C(G)_{k}$ for the special group $\Gamma(n, 2 n), n$ even and positive. Let $D$ be the following divisor on the Riemann surface of $G$ :

$$
D=k \operatorname{div}(\alpha)+\sum\left[k\left(1-1 / e_{i}\right)\right] w_{i}+\sum k p_{i}
$$

where the first summation runs through all elliptic points $w_{i}$ of period $e_{i}$ and the second summation runs through the parabolic points $p_{i}$, and where $\alpha$ denotes a differential. In [1, pp. 22-25] it is shown that $\operatorname{Dim} A(G)_{2 k}=\operatorname{dim} L(D)$ where $L(D)$ is the vector space of meromorphic functions on the Riemann surface whose divisors are $\geqslant-D$. Moreover, although not explicitly stated in [1, pp. 22-25], the same type of analysis gives $\operatorname{Dim} C(G)_{k}=\operatorname{Dim} L\left(D^{\prime}\right)$ where

$$
D^{\prime}=k \operatorname{div}(\alpha)+\sum\left[k\left(1-1 / e_{i}\right)\right] w_{i}+\sum(k-1) p_{i} .
$$

Then using the consequence of the Riemann-Roch theorem which asserts that if $A$ is a divisor such that $\operatorname{deg}(A)>2 g-2, g=$ genus, then

$$
\operatorname{Dim} L(A)=\operatorname{deg}(A)-g+1,
$$

and applying this fact to $A=D$ and then to $A=D^{\prime}$, we obtain, letting $\sigma(G)$ be the number of parabolic vertices of $G$ : 
If $k \geqslant 1, \operatorname{Dim} A(G)_{2 k}=(2 k-1)(g-1)+\sigma(G) k+\Sigma k\left(1-1 / e_{i}\right)$;

If $k=1, \operatorname{Dim} C(G)_{2 k}=g$ (assuming no elliptic points);

If $k>1, \operatorname{Dim} C(G)_{2 k}=(2 k-1)(g-1)+\sigma(G)(k-1)$.

For the case $G=\Gamma(n, 2 n)$ we know $g$ by $\S 1$. Moreover $\Gamma(n, 2 n), n$ even and positive, has no elliptic points because an elliptic transformation $(a z+b) /(c z+d)$ must satisfy $|a+d|<2, c \neq 0$. Finally, to compute $\sigma(G)$ we observe that $\sigma(G)$ for any group $G$ of finite index can be found as follows: Let $\Gamma(1)^{\prime}=\bigcup M_{i} G^{\prime}, 1 \leqslant i \leqslant t$, be a coset decomposition of $G^{\prime}$ in $\Gamma(1)^{\prime}$. Then $\sigma(G)$ is the number of distinct second rows in $M_{1}, \cdots, M_{t}$. In $\S 1$ we saw that $\left[\Gamma(n)^{\prime}: \Gamma(n, 2 n)^{\prime}\right]=4$. It is then easily seen that the coset decomposition $\Gamma(n, 2 n)^{\prime}$ in $\Gamma(n)^{\prime}$ has the following 4 matrices as a system of representatives:

$$
\left(\begin{array}{ll}
1 & 0 \\
0 & 1
\end{array}\right),\left(\begin{array}{ll}
1 & n \\
0 & 1
\end{array}\right),\left(\begin{array}{ll}
1 & 0 \\
n & 1
\end{array}\right),\left(\begin{array}{cc}
1+n^{2} & n \\
n & 1
\end{array}\right)
$$

Thus, if $\left(\begin{array}{ll}\boldsymbol{a} & \boldsymbol{b} \\ c & \boldsymbol{d}\end{array}\right)$ runs through a representative system for the cosets of $\Gamma(n)^{\prime}$ in $\Gamma(1)^{\prime}$ we get the following representative system for the cosets of $\Gamma(n, 2 n)^{\prime}$ in $\Gamma(1)^{\prime}$ :

$$
\begin{aligned}
\left(\begin{array}{ll}
1 & 0 \\
0 & 1
\end{array}\right)\left(\begin{array}{ll}
a & b \\
c & d
\end{array}\right) & =\left(\begin{array}{ll}
a & b \\
c & d
\end{array}\right), \\
\left(\begin{array}{ll}
1 & n \\
0 & 1
\end{array}\right)\left(\begin{array}{ll}
a & b \\
c & d
\end{array}\right) & =\left(\begin{array}{cc}
a+c n & b+n d \\
c & d
\end{array}\right), \\
\left(\begin{array}{ll}
1 & 0 \\
n & 1
\end{array}\right)\left(\begin{array}{ll}
a & b \\
c & d
\end{array}\right) & =\left(\begin{array}{cc}
a & b \\
c+a n & d+b n
\end{array}\right), \\
\left(\begin{array}{cc}
1+n^{2} & n \\
n & 1
\end{array}\right)\left(\begin{array}{ll}
a & b \\
c & d
\end{array}\right) & =\left(\begin{array}{cc}
\left(1+n^{2}\right) a+c n & \left(1+n^{2}\right) b+d n \\
n a+c & b n+d
\end{array}\right) .
\end{aligned}
$$

Observing that the second rows of the first 2 are the same and that the second rows of the last 2 are the same we conclude that the number of distinct second rows in the representative system for $\Gamma(n, 2 n)^{\prime}$ in $\Gamma(n)^{\prime}$ is twice that of $\Gamma(n)^{\prime}$ in $\Gamma(1)^{\prime}$. The latter is known to be $1 / 2 n^{2} \Pi\left(1-1 / p^{2}\right)$. Hence $\sigma(\Gamma(n, 2 n))=$ $n^{2} \Pi\left(1-1 / p^{2}\right)$. Putting together the above information gives:

THEOREM 1. Let $n$ be an even positive integer.

If $k \geqslant 1, \operatorname{Dim} A(\Gamma(n, 2 n))_{2 k}=((2 k-1) n+3) n^{2} \Pi\left(1-1 / p^{2}\right) / 6$.

If $k=1, \operatorname{Dim} C(\Gamma(n, 2 n))_{2 k}=n^{2}(n-3) \Pi\left(1-1 / p^{2}\right) / 6+1$.

If $k>1, \operatorname{Dim} C(\Gamma(n, 2 n))_{2 k}=((2 k-1) n-3) n^{2} \Pi\left(1-1 / p^{2}\right) / 6$.

3. Graded ring of theta constants. Let $e(z)$ denote $e^{2 \pi i z}$ and let $\tau$ denote a point in the upper half plane. Then for every $m=\left(m^{\prime}, m^{\prime \prime}\right)$ in $\mathbf{R}^{2}$ the series 


$$
\theta_{m}(\tau)=\sum_{p \in Z} e\left[\left(p+m^{\prime}\right) m^{\prime \prime}+1 / 2\left(p+m^{\prime}\right)^{2} \tau\right]
$$

defines a holomorphic function in $\tau$ called the theta constant of characteristic $m$. It is easily seen that for every $m$ in $\mathbf{R}^{2}$ and $n$ in $\mathbf{Z}^{2}$ we have

$$
\theta_{m}(\tau)=\theta_{-m}(\tau), \quad \theta_{m+n}(\tau)=e\left(m^{\prime} n^{\prime \prime}\right) \theta_{m}(\tau)
$$

Moreover, if $m$ is in $Q^{2}$ it is proved in [3, p. 227] that $\theta_{m}(\tau)$ vanishes identically if and only if $m \equiv(1 / 2,1 / 2) \bmod 1$. If $r$ is an even positive integer let $G_{r}$ denote the subgroup of the additive group $(Q / Z)^{2}$ consisting of those elements of order $r$. (In [3] the notation ${ }_{r}(\mathrm{Q} / \mathrm{Z})^{2}$ is used for $G_{r}$.) Consider the ring $\mathbf{C}\left[G_{r}\right]$ generated over $\mathbf{C}$ by all $\theta_{m}$ such that $m \bmod 1$ is in $G_{r}$. Then by the above $\mathrm{C}\left[G_{r}\right]$ is a ring of finite type. In fact, if we let $S_{r}$ denote the set of all $(t / r, s / r) \in(Q / Z)^{2}$ such that $0<t / r<1 / 2,0 \leqslant s / r<1$, or such that $t / r=1 / 2,0 \leqslant s / r<1 / 2$, or such that $t / r=0,0 \leqslant s / r \leqslant 1 / 2$, then by the above, $\mathbf{C}\left[G_{r}\right]=\mathbf{C}\left[\theta_{m}\right]$ where in the latter $m$ runs through all elements in $S_{r}$. Moreover, the cardinality of $S_{r}$ is given by

$$
\operatorname{Card}\left(S_{r}\right)=(r / 2-1) r+(r / 2)+(r / 2+1)=r^{2} / 2+1 .
$$

Now let $N(d, n)$ for the positive integers $n$ and $d$ denote the number of monomials of degree $d$ in $n$ letters. Also, let $N^{\prime}(d, n)$ denote the number of monomials of degree $d$ in $n$ letters such that each letter occurs at least once in the monomial. (Here we assume $d \geqslant n$.) Then simple combinatorial arguments yield

$$
\begin{aligned}
& N(d, n)=\left(\begin{array}{c}
n+d-1 \\
d
\end{array}\right)=\frac{(n-1+d) !}{(n-1) ! d !}, \\
& N^{\prime}(d, n)=\left(\begin{array}{c}
d-1 \\
d-n
\end{array}\right)=\frac{(d-1) !}{(d-n) !(n-1) !} .
\end{aligned}
$$

Thus the number of distinct monomials of degree $2 k, k \geqslant 1$, formed by considering each element of $S_{r}$ as a distinct letter is

$$
N\left(d, S_{r}\right)=\frac{\left(r^{2} / 2+2 k\right) !}{\left(r^{2} / 2\right) !(2 k) !}
$$

Now let $A\left[G_{r}\right], r$ even and positive, denote the subring $C\left[G_{r}\right]^{2}$ of $\mathbf{C}\left[G_{r}\right]$. That is, $\mathbf{C}\left[G_{r}\right]^{2}$ is the graded ring $\mathbf{C}\left[\theta_{m} \theta_{n}\right]$ where $m, n$ run through all elements of $S_{r}$, and where the $k$ th component of the grading is the vector space of homogeneous polynomials of degree $2 k$ in the theta constants $\theta_{m}$, $m \in S_{r}$. Thus, by the above

$$
\operatorname{Dim} A\left(G_{r}\right) \leqslant \frac{\left(r^{2} / 2+2 k\right) !}{\left(r^{2} / 2\right) !(2 k) !}
$$


Now $A\left(G_{r}\right)$ is a graded subring of the graded ring $A\left(\Gamma\left(r^{2}, 2 r^{2}\right)\right)=$ $\bigoplus \Sigma_{k \geqslant 0} A\left(\Gamma\left(r^{2}, 2 r^{2}\right)\right)_{k}$. Then a special case of a fundamental theorem in [3, p. 235] asserts that the integral closure of the graded ring $A\left(G_{r}\right), r$ an even positive integer, in its field of fractions is precisely $A\left(\Gamma\left(r^{2}, 2 r^{2}\right)\right)$. We can now answer Problem 1 of [3, p. 236] for the case $r=4$. (According to [4] this is already known.) We will do this by comparing the second component of the graded ring $A\left(G_{4}\right)$ to that of $A(\Gamma(16,32))$. By Theorem 1, $\operatorname{Dim} A(\Gamma(16,32))_{2}=$ 608 , and by the inequality of this section $\operatorname{Dim} A\left(G_{4}\right)_{2} \leqslant 495$. Thus $A\left(G_{4}\right)$ is properly contained in $A(\Gamma(16,32))$ and thus it is not integrally closed in its field of fractions.

4. Basis for cusp forms. Let $m=\left(m^{\prime}, m^{\prime \prime}\right)$ be in $\mathbf{R}^{2}$. If $M \in \Gamma(1)$, $M=\left(\begin{array}{ll}a & b \\ c & d\end{array}\right)$ define

$$
M m=\left(d m^{\prime}-c m^{\prime \prime},-b m^{\prime}+a m^{\prime \prime}\right)+1 / 2(c d, a b) .
$$

If $m \equiv n \bmod 1$ then $M m \equiv M n$ mod 1 and from this we see that the group $\Gamma(1)$ operates on the set $(R / Z)^{2}$. Moreover $G_{r}$ is stable, that is mapped to itself, by the action of $\Gamma(1)$. If $f$ is a holomorphic function in the upper half plane, $M$ an element of $\Gamma(1)^{\prime}, k$ a nonnegative integer, define $\left.f\right|_{k} M=$ $f(M(z))(c z+d)^{-k}$. Thus the condition that $f$ be in $C(G)_{k}$ is that $f$ be in $A(G)_{k}$ and that $\left.f\right|_{k} M$ has its 0 th Fourier coefficient equal to 0 , for each $M$ in $G^{\prime}$. Now consider the monomial $\Pi_{\alpha=1}^{2 k} \theta_{m_{\alpha}}, m_{\alpha} \in \mathbf{R}^{2}$. Then by the transformation law for theta constants [3, p. 226] we have

$$
\left.\prod_{\alpha=1}^{2 k} \theta_{m_{\alpha}}\right|_{k} M=C \prod_{\alpha=1}^{2 k} \theta_{M^{-1} m_{\alpha}}
$$

where $C$ is a nonzero constant depending only on $M, k, m_{\alpha}$. From now on we use the notation $\theta_{00}=\theta(00), \theta_{01 / 2}=\theta\left(0 \frac{1}{2}\right), \theta_{1 / 20}=\theta(1 / 20)$.

THEOREM 2. Given $f=\theta(00)^{n_{1}} \theta(01 / 2)^{n_{2}} \theta(1 / 20)^{n_{3}}$, where $n_{1}+n_{2}+n_{3}=$ $2 k_{0}$ Then $f$ is a cusp form for $\Gamma(4,8)$ of weight $k$ if and only if $n_{1}, n_{2}, n_{3}$, $>0$.

Proof. We define the type of a monomial $x^{n} y^{n_{2} z^{n_{3}}}$ to be the set $\left\{n_{1}, n_{2}, n_{3}\right\}$, where $n_{1}, n_{2}, n_{3}$ are not necessarily distinct, where some are possibly 0 , and where their ordering is irrelevant. (Thus $x^{2} y^{3} z^{2}$ and $x^{3} y^{2} z^{2}$ have the same type $(2,3,2)$.) We claim that a necessary and sufficient condition that 2 monomials $\theta(00)^{a} \theta\left(0 \frac{1}{b}\right)^{b} \theta(1 / 20)^{c}$ and $\theta(00)^{d} \theta\left(0 \frac{1}{2}\right)^{e} \theta(1 / 20)^{f}$ of the same degree $2 k$ have the same type is that there exists $M$ in $\Gamma(1)$ such that

$$
\left.\theta(00)^{a} \theta(01 / 2)^{b} \theta\left(\frac{1}{2} / 2\right)^{c}\right|_{k} M=\text { Const } \theta(00)^{d} \theta\left(0 \frac{1}{2}\right)^{e} \theta\left(1 \frac{1}{2} 0\right)^{f} \text {. }
$$


Sufficiency follows from the fact that since $\Gamma(1)$ operates on $G_{r}$ the induced mappings on $G_{r}$ must be injective. Necessity follows by observing that if

$$
M_{1}=\left(\begin{array}{ll}
1 & 0 \\
1 & 1
\end{array}\right), \quad M_{2}=\left(\begin{array}{rr}
-1 & -1 \\
3 & 2
\end{array}\right)
$$

and if

$$
g=(00), \quad h=(01 / 2), \quad i=(1 / 20)
$$

then

$$
\left(M_{1} g, M_{1} h, M_{1} i\right)=(i, h, g), \quad\left(M_{2} g, M_{2} h, M_{2} i\right)=(h, i, g)
$$

and hence

$$
\begin{aligned}
\left(M_{2}^{2} g, M_{2}^{2} h, M_{2}^{2} i\right) & =(i, g, h) \\
\left(M_{1} M_{2} g, M_{1} M_{2} h, M_{1} M_{2} i\right) & =(h, g, i) \\
\left(M_{2} M_{1} g, M_{2} M_{1} h, M_{2} M_{1} i\right) & =(g, i, h) .
\end{aligned}
$$

Thus $M_{1}, M_{2}, M_{2}^{2}, M_{1} M_{2}, M_{2} M_{1}, 1_{2}$ give all permutations of $(g, h, i)$ whence the sufficiency follows. Now a given monomial $f=\theta(00)^{a} \theta\left(0^{1 / 2}\right)^{b} \theta(1 / 20)^{c}$, $a+b+c=2 k$ is not a cusp form for $\Gamma(4,8)$ if and only if there exists $M$ in $\Gamma(1)$ such that the 0 th-Fourier coefficient $\left.f\right|_{k} M$ is not 0 . But the monomials with 0 th Fourier coefficient nonzero are precisely those of the form $\theta(00)^{j} \theta(01 / 2)^{k}, j \geqslant 0, k \geqslant 0$, as can be seen by the lemma below. At any rate, by this and the property above concerning type, we see that monomials which are not cusp forms are those of type $(a, b, c)$ where at least one of $a, b, c$ are 0 .

LEMMA. Let $r$ be an even positive integer and let $\left\{m_{\alpha}\right\}$ be $2 k$ characteristics such that $m_{\alpha} \bmod 1$ is in $G_{r}$. Then the Fourier expansion of $\Pi_{\alpha=1}^{2 k} \theta_{m_{\alpha}}$ with respect to the group $\Gamma\left(r^{2}, 2 r^{2}\right)$ is given by

$$
\prod_{\alpha=1}^{2 k} \theta_{m_{\alpha}}(\tau)=\sum_{\lambda=0}^{\infty} a_{\lambda} e\left(\lambda \tau / 2 r^{2}\right)
$$

where $a_{\lambda}=\Sigma e\left(\Sigma_{\alpha=1}^{2 k} x_{\alpha} m_{\alpha}^{\prime \prime} / r\right)$, the summation numing through all $\left(x_{1}, \cdots, x_{2 k}\right) \in$ $\mathrm{Z}^{2 k}$ satisfying $\Sigma_{\alpha=1}^{2 k} x_{\alpha}^{2}=\lambda, x_{\alpha} \equiv r m_{\alpha}^{\prime} \bmod r, 1 \leqslant \alpha \leqslant 2 k$.

Proof. Write the individual theta constant in the form

$$
\theta_{m}(\tau)=\sum_{p \in Z} e\left[\left(p+m^{\prime}\right) m^{\prime \prime}\right] e\left[\left(r p+r m^{\prime}\right)^{2} \tau / 2 r^{2}\right]
$$

The lemma follows by multiplying $2 k$ of these series.

Theorem 3. $A$ basis for the cusp forms in $A(\Gamma(4,8))_{2 k}, k \geqslant 1$, is given by the monomials $\theta(00)^{a} \theta\left(0 \frac{1}{2}\right)^{b} \theta(1 / 20)^{c}$ satisfying $a+b+c=4 k, a, b>0$, 
$1 \leqslant c \leqslant 4$. This statement holds for any permutation of $a, b, c$.

Proof. We are in the case $r=2$ for which it is known [2, p. 243] that $\mathrm{A}\left(G_{2}\right)=A(\Gamma(4,8))$. Let $x=\theta(00), y=\theta\left(0 \frac{1}{2}\right), z=\theta(1 / 20)$. Let $k=1$. Then $\operatorname{Dim} A(\Gamma(4,8))_{2}=14$ and $\operatorname{Dim} C(\Gamma(4,8))_{2}=3$. As there are only 15 monomials of degree 4 in the 3 theta constants and since $x^{4}=y^{4}+z^{4}$ by Riemann's theta formula it follows that $x y z^{2}, x y^{2} z, x^{2} y z$ are independent cusp forms and hence form the required basis. Now let $k>1$. Then the number of monomials in the statement is

$$
N^{\prime}(4 k-1,2)+N^{\prime}(4 k-2,2)+N^{\prime}(4 k-3,2)=16 k-4 .
$$

On the other hand $\operatorname{Dim} C(\Gamma(4,8))_{k}=16 k-14$. So there remains only to show that the monomials in the statement are linearly independent. From [2, p. 243] we know that

$$
A(\Gamma(4,8))=C[X, Y, Z] /\left[X^{4}-Y^{4}-Z^{4}\right] .
$$

Thus the only dependency relations are of the form

$$
g(x, y, z)=p(x, y, z)\left(x^{4}-y^{4}-z^{4}\right)
$$

where $g$ and $p$ are homogeneous polynomials of degree $4 k$ and $4 k-4$ respectively. Thus, $g(x, y, z)$ involves $z$ (also $x$ and $y$ ) to the degree $\geqslant 4$. Thus any collection $x^{a} y^{b} z^{c}, a+b+c=4 k, a, b>0,1 \leqslant c \leqslant 3$ is linearly independent. Moreover, if we throw into this set the monomials $x^{i} y^{j} z^{4}, i+j+$ $4=4 k, i, j>0$, then the entire set is linearly independent. Otherwise $g(x, y, z)=$ $p(x, y, z)\left(x^{4}-y^{4}-z^{4}\right)$ and since 4 is the highest power of $z$ in $g(x, y, z)$ we see that $p$ is a polynomial in $x$ and $y$ only. So the polynomials $p x^{4}$, $p y^{4}$ in $g$ do not involve $z$ and so the dependency relation is not among the monomials in the statement (which involve $z$ ).

Corollary 1. A basis for the cusp forms of weight $2 k, k \geqslant 1$, of the principal congruence group of level 4 is given by the set of monomials $\theta(00)^{2 a} \theta(0 / 2)^{2 b} \theta(1 / 20)^{2 c}$ satisfying $a+b+c=2 k, a, b>0, c=1$ or 2 . This statement holds for any permutation of $a, b, c$.

Proof. In the case $k=1$ there are no monomials of the stated form and $\operatorname{Dim} C(\Gamma(4))_{2}=$ genus $(\Gamma(4))=0$. This proves the case $k=1$. In the case $k>1$, it is known that $\operatorname{Dim} C(\Gamma(4))_{2 k}=4 k-5$. By [2, p. 243], $A(\Gamma(4))=$ $C\left[\theta(00)^{2}, \theta\left(0 \frac{1}{2}\right)^{2} \theta\left(\frac{1}{2} / 2\right)^{2}\right]$. By the theorem the monomials in the statement of the corollary are linearly independent and their numbers is just $N^{\prime}(2 k-1,2)+$ $N^{\prime}(2 k-2,2)=4 k-5$. Hence they form a basis.

COROLlaRY 2. $A$ basis for the cusp forms of weight $2 k, k>1$ of the 
principal congruence group of level 2 is given by the set of monomials $\theta(00)^{4 a} \theta\left(0 \frac{1}{2}\right)^{4 b} \theta\left(1 \frac{1}{2} 0\right)^{4}$ satisfying $a+b+1=k, a, b>0$.

Proof. If $k=1$ the above set is vacuous and also $\operatorname{Dim} C(\Gamma(2))_{2}=0$. If $k>1, \operatorname{Dim} C(\Gamma(2))_{4 k}=(2 k-1)$ (genus -1$)+\sigma(k-1)$ (where $\sigma=3$, genus $=0)=k-2$. But the number of monomials in the stated set is $N^{\prime}(k-1,2)=k-2$.

5. Application to Poincaré series. Let $\lambda \geqslant 1$ and for specificity denote the Fourier coefficient of $\Pi_{\alpha=1}^{2 k} \theta_{m_{\alpha}}$, whose expression is given in the lemma of $\S 3$, by $a_{\lambda}\left(\Pi_{\alpha=1}^{2 k} \theta_{m_{\alpha}}\right)$. Let $\varphi(\lambda, 2 k, G, \tau)$ denote the Poincaré series of weight $2 k$ and character $\lambda^{\alpha} \geqslant 1$ for a subgroup $G$ of $\Gamma(1)$ of finite index.

THEOREM 4. Let $G_{1}, G_{2}, G_{3}$ denote the groups $\Gamma(4,8), \Gamma(4)$ and $\Gamma(2)$ respectively. Let $S(1, k), S(2, k), S(3, k)$ denote the sets of monomials given in Theorem 3, Corollary 1 and Corollary 2, respectively, and let $f(1)=1, f(2)=$ $2, f(3)=4$. Then for $1 \leqslant i \leqslant 3, \lambda \geqslant 1$, the Poincaré series $\varphi\left(\lambda, 2 k, G_{i}, \tau\right)$ vanishes identically if and only if $a_{f(i) \lambda}\left(\Pi_{\alpha=1}^{4 k} \theta_{m_{\alpha}}\right)=0$ for every $\Pi_{\alpha=1}^{4 k} \theta_{m_{\alpha}}$ in $S(i, k)$.

Proof. This is a simple consequence of the fact that a Poincare series of character $\lambda \geqslant 1$ vanishes identically if and only if the $\lambda$ th Fourier coefficient of every cusp form vanishes.

COROllaRY 1. $\varphi(\lambda, 2 k, \Gamma(4), \tau)$ vanishes identically if $2 \lambda$ cannot be represented as $2 \lambda=x_{1}^{2}+\cdots+x_{4 k}^{2}$ with 2 or 4 of $x_{1}, \cdots, x_{2 k}$ congruent to $1 \bmod 4$ and the others congruent to $0 \bmod 4$.

COROllary 2. $\varphi(\lambda, 2 k, \Gamma(2), \tau)$ vanishes identically if $4 \lambda$ cannot be represented as $4 \lambda=x_{1}^{2}+\cdots+x_{4 k}^{2}$ with 4 of $x_{1}, \cdots, x_{2 k}$ congruent to $1 \bmod 4$ and the others congruent to $0 \bmod 4$.

\section{REFERENCES}

1. R. C. Gunning, Lectures on modular forms, Ann. of Math. Studies, no. 48, Princeton Univ. Press, Princeton, N. J., 1962. MR 24 \#A2664.

2. J.-I. Igusa, On the graded ring of theta-constants, Amer. J. Math. 86 (1964), 219246. MR $29 \# 2258$.

3. - On the graded ring of theta-constants. II, Amer. J. Math. 88 (1966), 221 236. MR $34 \# 375$.

4. - Geometric and analytic methods in the theory of theta-functions, Algebraic Geometry (Internat. Colloq., Tata Inst. Fund. Res., Bombay, 1968), Oxford Univ. Press, London, 1969, pp. 241-253. MR 42 \#6000.

DIVISION OF MATHEMATICAL SCIENCES, PURDUE UNIVERSITY, WEST LAFAYETTE, INDIANA 47907 